\title{
SIGNIFICADO PROGNÓSTICO DO LINFONODO METASTÁTICO N3 EM CARCINOMAS EPIDERMÓIDES DE CABEÇA E PESCOÇO
}

\author{
PROGNOSTIC SIGNIFICANCE OF N3 METASTATIC LYMPH NODE IN HEAD AND \\ NECK SQUAMOUS CARCINOMA
}

\author{
Ali Amar ${ }^{1}$ \\ Otávio Alberto Curioni ${ }^{1}$ \\ Abrão Rapoport, ECBC-SP ${ }^{1}$
}

\begin{abstract}
RESUMO: Objetivo: Avaliar os resultados do tratamento da doença metastática em estádio avançado (N3) e sua relação com o prognóstico do carcinoma espinocelular de cabeça e pescoço. Método: Foram revisados as informações de prontuários de 241 pacientes, com carcinoma espinocelular de boca, orofaringe, laringe e hipofaringe com metástases cervicais maiores que $6 \mathrm{~cm}(\mathrm{~N} 3)$ submetidos à cirurgia e/ou radioterapia, no Departamento de Cirurgia de Cabeça e Pescoço e Otorrinolaringologia do Hospital Heliópolis, Hosphel, São Paulo, de 1988 a 1998. Nos pacientes submetidos à cirurgia foi avaliada a radicalidade cirúrgica, macroscopicamente completa ou não, e naqueles tratados pela radioterapia, foi analisada a resposta do sítio primário e do pescoço imediatamente ao término do tratamento. A sobrevida livre de doença foi estimada pelo método de Kapplan Meier no grupo submetido à cirurgia. Resultados: A irressecabilidade da lesão primária e metastática no pescoço justificou a indicação da radioterapia na dose média de $65 \mathrm{~Gy}$, em 69 pacientes ocorrendo resposta completa no sítio primário em 24(36\%), no pescoço em 12 (18\%), e em ambos os sítios em 11 casos $(16 \%)$. No grupo sumetido à cirurgia seguido de radioterapia, a dose média foi de $56 \mathrm{~Gy}$. Dos 25 pacientes com ressecção macroscópica radical do pescoço, cinco (20\%) recidivaram, e dos cinco com ressecção incompleta e radioterapia, dois tiveram sobrevida de sete a 12 meses após o tratamento, quando foram perdidos de seguimento. A sobrevida livre de doença em dois anos neste grupo foi de 58\%. Conclusões: Para pacientes com linfonodo metastático N3, o esvaziamento cervical seguido de radioterapia foi eficiente no controle regional da doença enquanto que nos inoperáveis, a radioterapia é um tratamento paliativo.
\end{abstract}

Descritores: Linfonodo; Metástase neoplásica; Carcinoma de células escamosas; Neoplasias de cabeça e pescoço; Prognóstico.

\section{INTRODUÇÃO}

A presença de metástases linfáticas é um dos marcadores prognósticos mais relevantes para os pacientes com carcinoma das vias aerodigestivas superiores, pois está relacionada ao aumento significativo nas taxas de recidiva loco-regional e metástases à distância ${ }^{1}$.

Um dos princípios do tratamento cirúrgico das neoplasias malignas consiste na ressecção macroscópica completa do tumor, um objetivo difícil de ser atingido quando a doença engloba diversas estruturas nobres. Além disto, as metástases volumosas estão associadas a sítios primários com baixos índices de controle local, como a orofaringe e a hipofaringe. O esvaziamento cervical é um tratamento eficiente no controle regional da doença, embora não tenha a mesma repercussão nas taxas de sobrevida. O controle loco-regional pode representar um ganho em qualidade de vida, porém, a cirurgia higiênica não é habitualmente considerada nestes pacientes, e um dos motivos é o longo intervalo de tempo despendido no tratamento e na reabilitação, associado à precocidade da recidiva após uma ressecção incompleta do tumor. Quando a doença regional avançada está associada à doença igualmente extensa no

1. Cirurgião do Departamento de Cirurgia de Cabeça e Pescoço e Otorrinolaringologia do Hospital Heliópolis, Hosphel, São Paulo.

Recebido em 18/12/2002

Aceito para publicação em 29/04/2003

Trabalho realizado pelos Departamentos de Cirurgia de Cabeça e Pescoço e Otorrinolaringologia do Hospital Heliópolis/SP. 
sítio primário, raros pacientes são candidatos a tratamento com intenção curativa. Nesta situação, a morbimortalidade do tratamento cirúrgico e a remota possibilidade de cura remetem à outras modalidades terapêuticas. Quando a doença regional extensa está associada a tumores primários pequenos ( $\mathrm{T} 1 \mathrm{ou} \mathrm{T} 2)$ ou mesmo tumor primário oculto, deve ser contemplada a proposta de esvaziamento cervical.

Este estudo tem por objetivo avaliar os resultados do tratamento da doença metástatica cervical em estádio avançado (N3), e sua relação com o prognóstico do carcinoma espinocelular de cabeça e pescoço.

\section{MÉTODO}

Foram revisadas as informações dos prontuários dos pacientes atendidos no Departamento de Cirurgia de Cabeça e Pescoço e Otorrinolaringologia do Hospital Heliópolis entre janeiro de 1988 e dezembro de 1998. Foram atendidos 241 pacientes com carcinoma epidermóide de boca, orofaringe, laringe e hipofaringe com metástases cervicais maiores que 6 $\mathrm{cm}$ (N3), submetidos à cirurgia ou radioterapia. Destes, 142 pacientes não retornaram após a primeira consulta e foram excluídos, restando 99 pacientes que foram agrupados conforme a modalidade terapêutica principal: cirurgia ou radioterapia. As características dos pacientes quanto à idade, sexo, sítio primário e estádio T são demonstradas na Tabela 1, e avaliado o

Tabela 1 - Características dos pacientes.

\begin{tabular}{lcc}
\hline & Radioterapia & Cirurgia \\
\hline Idade média & 56 & 57 \\
Sexo & & \\
Masculino & 68 & 28 \\
Feminino & 1 & 2 \\
Sítio Primário & & \\
Boca & 14 & 4 \\
Orofaringe & 34 & 12 \\
Laringe & 9 & 3 \\
Hipofaringe & 12 & 11 \\
Estádio T & & \\
Tx & 0 & 1 \\
T0 & 0 & 2 \\
T1 & 4 & 2 \\
T2 & 8 & 9 \\
T3 & 15 & 14 \\
T4 & 42 & 2 \\
\hline
\end{tabular}

controle loco-regional da doença, o desenvolvimento de metástase à distância e segundo tumor. Nos pacientes submetidos à cirurgia foi avaliada a radicalidade da ressecção do tumor, macroscopicamente completa ou incompleta.

A sobrevida livre de doença foi estimada pelo método de Kaplan-Meier. Nos pacientes submetidos à radioterapia foi considerado o motivo desta opção terapêutica e avaliada a resposta no sítio primário e no pescoço imediatamente após o término do tratamento.

\section{RESULTADOS}

\section{Radioterapia}

O principal motivo para indicação de radioterapia exclusiva ou associada à quimioterapia foi a irressecabilidade da lesão (Figura 1) com dose média de 65 Gy (30 a 82 Gy). Treze pacientes receberam tratamento quimioterápico neoadjuvante. Apenas quatro pacientes $(5 \%)$ permaneceram com a doença controlada por mais de 12 meses. Entre os 69 pacientes irradiados, 66 apresentavam registro da resposta ao tratamento em ambos os sítios (primário e pescoço). A resposta completa no sítio primário foi observada em 24 (36\%) pacientes e no pescoço em 12 (18\%) (Tabela 2). A resposta completa em ambos os sítios foi observada em 11 pacientes (16\%), entre os quais quatro foram perdidos de seguimento nos primeiros cinco meses e cinco apresentaram recidivas em um intervalo de quatro a 23 meses após o início do tratamento. Um destes foi resgatado com cirurgia. Dos 58 pacientes remanescentes que apresentavam doença persistente ou resposta não avaliada, 16 foram acompanhados até o óbito, que ocorreu em média 240 dias após o início do tratamento.As metástases à distância foram diagnosticadas em quatro pacientes, além dos três que já as apresentavam no início do tratamento.

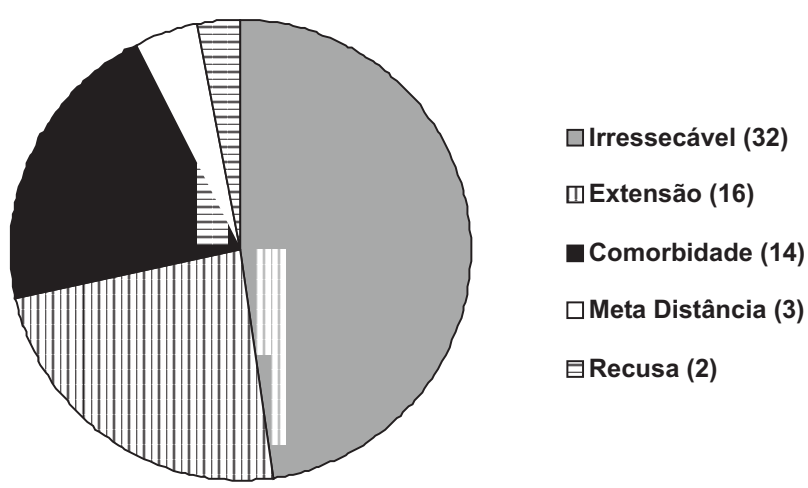

Figura 1 - Motivo do tratamento não cirúrgico. 
Tabela 2 - Resposta ao tratamento radioterápico no sítio primário e no pescoço.

\begin{tabular}{llcccc} 
& \multicolumn{2}{c}{ Pescoço } \\
\cline { 2 - 6 } PRIMÁRIO & Nx & Sem resposta & Completa & Parcial & Total \\
\hline Tx & 4 & 2 & 0 & 0 & $\mathbf{6}$ \\
Sem resposta & 0 & 27 & 1 & 1 & $\mathbf{2 9}$ \\
Completa & 1 & 8 & 11 & 4 & $\mathbf{2 4}$ \\
Parcial & 0 & 2 & 0 & 5 & $\mathbf{7}$ \\
Total & $\mathbf{5}$ & $\mathbf{3 9}$ & $\mathbf{1 2}$ & $\mathbf{1 0}$ & $\mathbf{6 6}$ \\
\hline
\end{tabular}

\section{Cirurgia}

Entre os 30 pacientes submetidos à cirurgia, 24 fizeram radioterapia pós-operatória com dose média de 56 Gy (50 a 70 Gy). Cinco pacientes tiveram a cirurgia abortada devido à impossibilidade de ressecção completa do tumor no pescoço, ocorrendo um óbito pós-operatório.

Dos 25 pacientes com ressecção macroscópica completa no pescoço, cinco (20\%) pacientes apresentaram recidiva cervical, em um a recidiva foi contralateral e resgatada com sucesso. Entre os cinco pacientes com ressecção incompleta e submetidos à radioterapia pós-operatória, dois apresentavam doença controlada aos sete e 12 meses após o tratamento, quando foram perdidos de seguimento. As metástases à distância foram diagnosticadas em dois pacientes e outros dois pacientes desenvolveram segundo tumor primário. A sobrevida livre de doença em dois anos foi de $58 \%$ neste grupo (Figura 2).

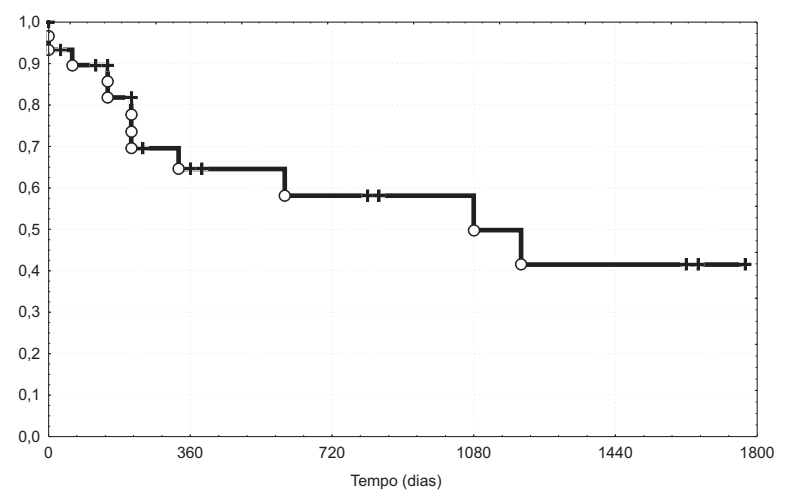

Figura 2 - Sobrevida livre de doença nos pacientes operados.

\section{DISCUSSÃO}

O tratamento combinado, com cirurgia seguida de radioterapia, apresenta as melhores pos- sibilidades de controle da doença em estádio avançado, porém muitos pacientes não apresentam condições para esta abordagem terapêutica. Os resultados observados nos pacientes submetidos à radioterapia exclusiva demonstram que este tratamento é insuficiente na presença de metástases volumosas no pescoço (N3) ${ }^{2}$. Há uma maior probabilidade de resposta do tumor primário ao tratamento irradiante, em comparação às metástases cervicais. Mesmo com resposta completa no pescoço, a possibilidade de doença residual é alta e deve ser considerado o esvaziamento cervical sempre que possível. Narayan et al. ${ }^{3}$ reportam doença residual em $62 \%$ dos espécimes de esvaziamento pós-radioterapia em pacientes cujas metástases eram inicialmente maiores do que 4 $\mathrm{cm}$. A indicação de esvaziamento cervical, nesta situação, também se justifica pela remota possibilidade de resgate de uma recidiva, porém exige que a doença esteja controlada no sítio primário. A resposta ao tratamento radioterápico pode ser difícil de determinar ao término do tratamento, quando as alterações teciduais causadas pela irradiação e o processo cicatricial dificultam a detecção de doença residual. Os métodos de imagem convencionais são imprecisos e as biópsias em múltiplos pontos devem ser realizadas. A tomografia por emissão de pósitrons (PET scan) é o método de escolha, mas não está disponível para uso rotineiro em nosso meio ${ }^{4}$.

A quase totalidade dos pacientes submetidos ao tratamento radioterápico exclusivo ou precedido de quimioterapia não apresentavam condições para a realização de cirurgia, tanto pela extensão da doença no sítio primário ou no pescoço, como também pela falta de condições clínicas para realização de um procedimento prolongado. Aproximadamente $30 \%$ destes pacientes apresentaram resposta parcial ou completa ao término da irradiação. Diante dos maus re- 
sultados observados com a radioterapia exclusiva, alguns destes pacientes podem ser candidatos a uma cirurgia de resgate, especialmente aqueles com tumor primário inicialmente pequeno (T1 ou T2). Diante de um tumor primário previamente irressecável, a realização do esvaziamento cervical pode ser um procedimento fútil.

Uma abordagem terapêutica de exceção pode ser empregada em um tumor primário pequeno com metástase volumosa, em que o tratamento irradiante do sítio primário (preservação de órgãos) é antecedido pelo esvaziamento cervical. Esta metodologia fere um princípio do tratamento oncológico, ou seja, o tratamento do pescoço sem o controle da doença no sítio primário, porém, a abordagem do pescoço antes da realização da radioterapia apresenta vantagens técnicas.

Smeele et al..$^{5}$ avaliaram os esvaziamentos com margem cirúrgica comprometida e relatam taxas de controle cervical de até $75 \%$ em dois anos, embora a sobrevida doença-específica no mesmo período seja de apenas $33 \%$. Os resultados são semelhantes aos do presente estudo e refletem as recidivas em outros sítios (primário e à distância). De fato, a incidência de metástases à distância é diretamente proporcional ao estadiamento do pescoço, atingindo aproximadamente $35 \%$ no pescoço N3 ${ }^{6,7}$. As metástases à distância foram diagnosticadas em $10 \%$ dos pacientes, refletindo um provável subdiagnóstico causado pela elevada perda de seguimento como também pela limitação da investigação diagnóstica em pacientes com prognóstico fechado.

Dentre as estruturas anatômicas não ressecadas durante o esvaziamento, a artéria carótida é a que mais freqüentemente apresenta comprometimento ou suscita dúvida quanto à ressecção completa do tumor ${ }^{5}$. A parede arterial e a bainha carotídea usualmente atuam como barreira à invasão tumoral, mas as lesões avançadas no pescoço podem aderir e invadir a artéria carótida, especialmente após o tratamento irradiante. Cerca de 5 a $20 \%$ dos pacientes submetidos a esvaziamento cervical tem algum envolvimento da artéria carótida ${ }^{8,9}$. Este envolvimento da carótida por uma metástase linfonodal não apenas indica um pior prognóstico, como também uma potencial contra-indicação à ressecção cirúrgica. O envolvimento da carótida parece ser particularmente comum nos casos recidivados. As metástases maiores que $6 \mathrm{~cm}(\mathrm{~N} 3)$ raramente estão confinadas no linfonodo. Quando o envolvimento extranodal ocorre em direção ao músculo esternocleidomastoideo e à pele, a neoplasia ainda pode ser radicalmente removida, entretanto, as metástases volumosas crescem em todas as direções e as primeiras estruturas a serem invadidas são a veia jugular interna, a carótida, os músculos pré-vertebrais e a base do crânio. Se a remoção do tumor for incompleta, raramente será obtido o controle regional da doença, independentemente da associação terapêutica empregada. Por muito tempo as metástases que invadiam a carótida foram consideradas irressecáveis. Todavia, o critério de ressecabilidade destas lesões tem se alterado nos últimos anos, em conseqüência da evolução técnica, mas continua a provocar controvérsias devido à significativa morbi-mortalidade e aos baixos índices de sobrevida ${ }^{10,11}$. A ressecção da subadventícia é considerada paliativa, pois freqüentemente é incompleta, e também coloca o vaso em risco de ruptura, seja pelo adelgaçamento da parede, crescimento do tumor residual ou efeito da radioterapia. A ressecção completa é a única abordagem que pode proporcionar o controle regional da doença. A ressecção da artéria carótida, quando necessária, somente deve ser indicada em casos bem selecionados, em pacientes com bom estado geral, com grande probabilidade de controle no sítio primário e possibilidade de ressecção macroscópica completa do tumor no pes$\operatorname{coço}^{12}$.

A maioria dos pacientes submetidos à radioterapia não apresentou resposta, especialmente no pescoço. Assim como nos protocolos de preservação de órgãos, deve ser questionada a continuidade do tratamento na ausência de resposta após uma dose de aproximadamente $45 \mathrm{~Gy}$, quando os efeitos deletérios da irradiação se somam aos sintomas da doença.

Em conclusão, os pacientes com metástases cervicais em estádio avançado (N3) apresentam um péssimo prognóstico, porém, o esvaziamento cervical seguido de radioterapia é eficaz no controle regional da doença. Nos pacientes inoperáveis, a radioterapia na maioria das vezes constitui um tratamento paliativo. 


\begin{abstract}
Background: We assessed the results of therapy for advanced lymph node metastases (N3) and its relation with the prognosis of squamous cell carcinoma of the head and neck. Methods: Files of 241 patients with squamous cell carcinoma of the mouth, oropharynx, larynx and hipopharynx with advanced metastatic lymph nodes (N3), submited to surgery and/or radiotherapy, were reviewed at the Head and Neck and Otorhinolaryngology Department of Hospital Heliópolis, Hosphel, São Paulo (1988 to 1998). For the surgical group, complete or incomplete resection was evaluated and for the irradiated group the responsiveness at the end of the therapy was analysed. Disease-free survival for the first group was evaluated using Kaplan-Meier. Results: The unresecability of the neoplasia justified the radiotherapy at medium dose of 65 Gy for 69 patients, with complete response at primary lesion in 24(36\%), and at the neck in 12(18\%), and for both sites in 11(16\%). For the surgical group followed by irradiation, the medium dose was 56 Gy. From 25 patients submitted to radical resection of the neck, five (20\%) presented recurrence, and the five cases with partial resection and radiotherapy, two presented a global survival from seven to 12 mouths. For the surgical group, disease-free survival for two years was 58\%. Conclusions: For patients with N3 neck node metastases, neck dissection, followed by radiotherapy, was effective for disease regional control, while, for inoperable cases, radiotherapy was only a paliative method.
\end{abstract}

Key Words: Lymph Node; Neoplasm metastasis; Carcinoma, squamous cell; Head and Neck neoplasms; Prognosis.

\section{REFERÊNCIAS}

1. Myers EN, Fagan JJ - Treatment of the N+ neck in squamous cell carcinoma of the upper aerodigestive tract. Otolaryngol Clin North Am, 1998, 31(4):671-686.

2. Narayan K, Crane CH, Kleid S, et al. - Planned neck dissection as an adjunct to the management of patients with advanced neck disease treated with definitive radiotherapy: for some or for all? Head Neck, 1999, 21(7):606-613.

3. Grandi C, Mingardo M, Guzzo M, et al. - Salvage surgery of cervical recurrences after neck dissection or radiotherapy. Head Neck, 1993, 15(4):292-295.

4. Wong RJ, Lin DT, Schöder H, et al. - Diagnostic and prognostic value of [(18)F]fluorodeoxyglucose positron emission tomography for recurrent head and neck squamous cell carcinoma. J Clin Oncol, 2002, 20(20):4199-4208.

5. Smeele LE, Leemans CR, Langendijk JA, et al. - Positive surgical margins in neck dissection specimens in patients with head and neck squamous cell carcinoma and the effect of radiotherapy. Head Neck, 2000, 22(6):559-563.

6. Suárez C, Llorente JL, Nuñez F, et al. - Neck dissection with or without postoperative radiotherapy in supraglottic carcinomas. Otolaryngol Head Neck Surg, 1993, 109(1):3-9.
7. Spector JG, Sessions DG, Haughey BH, et al. - Delayed regional metastases, distant metastases, and second primary malignancies in squamous cell carcinomas of the larynx and hypopharynx. Laryngoscope, 2001, 111(6):1079-1087.

8. Kennedy JT, Krause CJ, Loevy S - The importance of tumour attachment to the carotid artery. Arch Otolaryngol, 1977, 103(2):70-73.

9. Suárez Nieto C, Estevan Solano JM, Buron Martinez $\mathrm{G}$, et al. - Invasion of the carotid artery in tumours of the head and neck. Clin Otolaryngol, 1981, 6(1):29-37.

10. Meleca RJ, Marks SC - Carotid artery resection for cancer of the head and neck. Arch Otolaryngol Head Neck Surg, 1994, 120(9):974-978.

11. Nishinari K, Wolosker N, Yazbek G, et al. - Carotid reconstruction in patients operated for malignant head and neck neoplasia. Sao Paulo Med J, 2002, 120(5):137140.

12. Galati LT, Johnson JT - Management of advanced neck disease. Rev Bras Cir Cabeça Pescoço, 1998, 22(1):7-8.

Endereço para correspondência:

Prof. Dr. Abrão Rapoport

Praça Amadeu Amaral, 47 - cj. 82 - Paraíso

01327-010 - São Paulo - SP

E-mail: cpgcp.hosphel@attglobal.net 